\title{
Hand hygiene technique quality evaluation in nursing and medicine students of two academic courses ${ }^{1}$
}

\author{
Manuela Škodová ${ }^{2}$ \\ Alfredo Gimeno-Benítez ${ }^{3}$ \\ Elena Martínez-Redondo ${ }^{4}$ \\ Juan Francisco Morán-Cortés ${ }^{5}$ \\ Ramona Jiménez-Romano ${ }^{6}$ \\ Alfredo Gimeno-Ortiz ${ }^{6}$
}

\begin{abstract}
Objective: because they are health professionals, nursing and medical students' hands during internships can function as a transmission vehicle for hospital-acquired infections. Method: a descriptive study with nursing and medical degree students on the quality of the hand hygiene technique, which was assessed via a visual test using a hydroalcoholic solution marked with fluorescence and an ultraviolet lamp. Results: 546 students were assessed, 73.8\% from medicine and $26.2 \%$ from nursing. The area of the hand with a proper antiseptic distribution was the palm (92.9\%); areas not properly scrubbed were the thumbs (55.1\%). $24.7 \%$ was very good in both hands, $29.8 \%$ was good, $25.1 \%$ was fair, and $20.3 \%$ was poor. The worst assessed were the male, nursing and first year students. There were no significant differences in the age groups. Conclusions: hand hygiene technique is not applied efficiently. Education plays a key role in setting a good practice base in hand hygiene, theoretical knowledge, and in skill development, as well as good practice reinforcement.
\end{abstract}

Descriptors: Students; Medicine; Nursing; Hand Disinfection; Evaluation.

\footnotetext{
${ }^{1}$ Paper extracted from doctoral dissertation "Evaluación de la Calidad de la Técnica de Higiene de Manos en los Profesionales Sanitarios de Complejo Hospitalario Universitario Infanta Cristina de Badajoz y en los Estudiantes de Grado de Enfermería y Grado de Medicina del Campus Universitario de Badajoz de la Universidad de Extremadura, periodo de 2012 a 2014", presented to Universidad de Extremadura, Badajoz, Extremadura, Spain.

2 Doctoral student, Faculdad de Medicina, Universidad de Extremadura, Badajoz, Extremadura, Spain. Associate Professor, Servicio de Medicina Preventiva, Hospital Infanta Cristina, Badajoz, Spain.

3 PhD, Associate Professor, Departamento de Enfermería, Universidad de Extremadura, Badajoz, Extremadura, Spain.

4 Master's student, Faculdad de Medicina, Universidad de Extremadura, Badajoz, Extremadura, Spain. RN, Servicio Extremeño de Salud, Badajoz, Extremadura, Spain.

5 MSc, RN, Servicio Extremeño de Salud, Badajoz, Extremadura, Spain.

${ }_{6}$ PhD, Associate Professor, Servicio de Medicina Preventiva, Hospital Infanta Cristina, Badajoz, Extremadura, Spain.
}

Corresponding Author:

Juan Francisco Morán Cortés

Universidad de Extremadura. Departamento de Enfermería

C/ Ordesa, 5

06006, Badajoz, Extremadura, España

E-mail: juanfmoran@gmail.com
Copyright (๑) 2015 Revista Latino-Americana de Enfermagem This is an Open Access article distributed under the terms of the Creative Commons Attribution Non-Commercial License (CC BY-NC).

This license lets others distribute, remix, tweak, and build upon your work non-commercially, and although their new works must also acknowledge you and be non-commercial, they don't have to license their derivative works on the same terms. 


\section{Introduction}

Hospital-acquired infections (HAIs) are one of the main causes for morbility and mortality in the health field, which constitute one of the main issues in global public health(1).

Health professionals' hands are one of the main transmission mechanisms for HAIs. Hand washing with water and antiseptic soap before and after patient contact is the most efficient technique proven to prevent hospital-acquired infection (2).

However, in everyday clinical practice, hand hygiene $(\mathrm{HH})$ is happening less often than desired(3).

The World Health Organizations' (WHO) recommendations about enhancement strategies and better $\mathrm{HH}$ practice are considered as reference criteria, setting up several educational interventions targeting health professionals(4).

Both in Spain ${ }^{(5)}$ and in the Autonomous Community of Extremadura(6), promotion and knowledge development as well as a culture of patient safety are being stressed among professionals and patients in all health service levels. While performing its working lines on a local stage, the Complejo Hospitalario Universitario Infanta Cristina de Badajoz, the Sociedad Española de Medicina Preventiva, Salud Pública e Higiene (SEMPSPH) planned educational seminars and workshops about hand hygiene and its assessment.

Because they are health professionals, nursing and medical students' hands during internships can function as a transmission vehicle for hospital-acquired infections, and can cause patient, object and surface contamination $^{(7)}$.

In this study we plan to assess the current state of $\mathrm{HH}$ in nursing and medicine students, enrolled to the Facultad de Medicina del Campus de Badajoz of the Universidad de Extremadura (UEX), who were doing an internship at the Complejo Hospitalario Universitario Infanta Cristina de Badajoz (CHUICB).

\section{Method}

Our study was a descriptive, cross-sectional study that occurred in two periods of time, and a sample was limited by the UEX, namely the Medicine Campus where medicine (six courses) and nursing (four courses) undergraduate studies are available. Three hundred seventeen students were enrolled in the nursing degree 2012/13 class, and 294 students in the 2013/14 class. For the medicine degree, there were 877 students for the 2012/13 class and 878 for the $2013 / 14$ class.

The CHUICB is integrated with the Hospital Infanta Cristina, Hospital Perpetuo Socorro, Hospital MaternoInfantil and the Specialty Center. This complex belongs to the Health Department of Badajoz, which served a populace of 276, 154 people; it owned 831 beds, had a total of 40, 434 hospital admissions, 31, 533 surgical procedures, 2,430 deliveries and the mean stay was 6.84 days $^{(8)}$.

No selection of the student' sample was conducted. All students attending preventive medicine and public health classes of the biomedical sciences department and community nursing I and II classes of the nursing department were included. Student participation was voluntary.

Nursing and medical students from the Medicine Campus of Badajoz who participated in our study were: nursing degree students in the second and third years, medicine degree students in second and fifth year, and medicine baccalaureate students in sixth year (last class of the old program).

The study occurred in two periods of time: Academic year 2012/213 and 2013/2014

The study was conducted by the same professionals in the preventive medicine and public health service, on several days and different schedules in order to study the whole sample of students. A one-hour theory lesson about the foundations of hand, object, and surface contamination, epidemiology on the chain of bacteria transmission, and the different kinds of $\mathrm{HH}$ (instructions, material and technique) were taught during the school year of 2012/13 and 2013/14. The lesson focused on hygienic hand washing, antiseptic hand washing and hand rubbing with hydroalcoholic solutions. Likewise, instructions on applying $\mathrm{HH}$, following the methodology of the "five moments of hand hygiene" proposed by the WHO were stressed.

During practical teaching, nursing and medical students attended a simulated specialty medical practice session. Small groups were established with five students. The reason for visit was explained (nausea) and students were asked to care for the patient (taking vital signs); asking them to perform a correct $\mathrm{HH}$ following WHO commendations. There was no sink or water and soap for performance of the $\mathrm{HH}$, only hydroalcoholic solution was available which students had to use, applying knowledge acquired in the theoretical class.

Identifying variables included: date, center, academic course, nursing or medicine, sex and age. 
An alcohol-based mix marked with fluorescence and an ultraviolet (UV) lamp (Dermalux $®$, Derma LiteCheck by Dermalux - Training) were used to assess $\mathrm{HH}$.

A visual assessment of the correct fluorescencemarked hydroalcoholic solution (HAS) distribution (categories yes/no) was performed. Five main sections were considered: palms, back of the hand, between the fingers, finger tips/nails separately for each hand (right and left) and for both hands.

For the final quality assessment of the $\mathrm{HH}$ technique, some categories were established: "very good" if HAS was spread throughout all sections, "good", if four sections were exposed, "fair" if two sections were not exposed, and "poor" if three or more regions were left without HAS exposure (Likert-type scale with four categories). Subsequently, they were divided in two categories: "proper $\mathrm{HH}^{\prime \prime}$ when the right hand, left hand and both hands obtained a "very good" or "good"

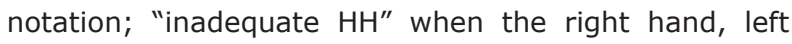
hand or both hands obtained a "fair" or "poor" notation.

Limitations to the study included: lack of a randomized sample, as well as the concomitant differences in year of education, which could bias the study.

A separate descriptive analysis of the variables was conducted, presenting the mean corresponding to the qualitative variables, and centralizing measures as well as dispersion of the quantitative variables.

A chi-square $(x 2)$ was used for the bivariant analyses of the qualitative variables and a Student t-test for the quantitative variables, considering as significative the values $p>0.05$.

Excel of Microsoft Office 2007 was used for the coding of the obtained data, and SPSS version 15.0 for the statistical analysis.

Ethical factors: Participation of all subjects in the study was voluntary. Confidentiality of data (Organic Law 15/1999, of December 13, of the Protection of Personal Character Data) and statistics (group coding, analysis and results) were kept secret at all times; likewise, the compliance was maintained with the Hospital Infanta Cristina de Badajoz's (Spain) Ethics Committee's research protocols.

\section{Results}

A total of 546 students participated in the study, 403 (73.8\%) of them were medical students and $143(26.2 \%)$ were nursing students; 216 (39.6\%) students were from the $2012 / 2013$ class and $330(60.4 \%)$ students were from the 2013/2014 class. Males accounted for $30.45 \%$
(144), and 69.6\% (380) were female. The mean age of the sample was $21.4 \pm 3.73$ years of age.

In general, HAS distribution on the right hand was correct in $96.5 \%$ of cases on the palm, $86.1 \%$ between the fingers, $72.7 \%$ on the back of the hand, $70.3 \%$ on the finger tips, and $56.9 \%$ on the thumbs. For the left hand: $95.2 \%$ on the palm, $82.6 \%$ between the fingers, $80.4 \%$ on the back of the hand, $68.7 \%$ on the finger tips, and $63 \%$ on the thumbs. Considering both hands, th eHAS covered: $92.9 \%$ on the palms, $78.02 \%$ between the fingers, $65.2 \%$ on the finger tips, $64.2 \%$ on the back of the hand, and $55.1 \%$ on the thumbs.

Through direct observation, right hand, left hand and both hand $\mathrm{HH}$ technique quality was obtained. It was noted that $34.1 \%$ performed $\mathrm{HH}$ on the right hand by spreading HAS on five sections properly, $29.5 \%$ performed good $\mathrm{HH}, 21.7 \%$ achieved a fair score, and $14.6 \%$ achieved a poor score. For the left hand, 38.5\% obtained a very good $\mathrm{HH}$ score, $30.9 \%$ had one mistake a $19.9 \%$ had two mistakes, $20.4 \%$ had three or more mistakes. Thus, $24.7 \%$ in both hands was very good, $29.8 \%$ was good, $25.1 \%$ was fair, and $20.3 \%$ was poor.

Category results were as follows: right hand $\mathrm{HH}$ was appropriate in $63.5 \%, 69.4 \%$ on left hand and $\mathrm{HH}$ for both hands was accurate in $50.2 \%$ of the students.

In terms of bivariant analysis by sex, men spread HAS worse than women in between the fingers and the back of the hand, on both the right and left hand (table 1). Observation for both hands showed that men did not spread HAS to the thumbs and in between the fingers as often as women did. Likewise, it was the men who obtained a "fair" notation on the right hand and "poor" on both hands, with significant differences versus women. These differences kept grouping the evaluation into two $\mathrm{HH}$ categories, which were: inappropriate $\mathrm{HH}$ on the right hand, and both hands, for men (table 1 ). There were no difference in the men and women groups based on year, course or age.

Table 2 shows that nursing students performed inappropriate $\mathrm{HH}$ on the right hand 2.2 times and on the left hand 1.7 times $(p<0,05)$ more often than medical students. Future nurses obtained a "fair" and "poor" notation on the right hand and "poor" on the left hand, with significant differences compared to the medical students. Hand sections most often left without HAS by nursing students versus medical students were the palm, thumb and in between fingers of right hand; back of the hand and between the fingers on left hand, leaving back of hands, thumbs and in between fingers poorly washed on both hands (Table 2, p<0,05). 
Hand hygiene technique quality was significatively better for the 2013/14 class versus the previous class and in women; there were no differences per year of study or age (Table 3). Table 3 shows how a lack of rubbing HAS in between the fingers and thumbs stood out as a factor most involved in inappropriate $\mathrm{HH}$.

Table 1 - HAS spreading on students' hands as per sex, marked section and degree of sanitation. Facultad de Medicina de Badajoz. Badajoz. Spain. 2012/2014

\begin{tabular}{|c|c|c|c|c|c|c|c|c|}
\hline \multirow{2}{*}{ Sections } & & \multirow{2}{*}{ Value } & \multicolumn{2}{|c|}{ Male } & \multicolumn{2}{|c|}{ Female } & \multirow{2}{*}{ OR } & \multirow{2}{*}{$\mathrm{Cl} 95 \%$} \\
\hline & & & $\mathbf{N}$ & $\%$ & $\mathbf{N}$ & $\%$ & & \\
\hline \multirow[t]{4}{*}{ Palm } & Right & No & 7 & 4.2 & 12 & 3.2 & 1.35 & $0.52-3.49$ \\
\hline & & Yes & 159 & 95.8 & 368 & 96.8 & & \\
\hline & Left & No & 12 & 7.2 & 14 & 3.7 & 2.04 & $0.92-4.51$ \\
\hline & & Yes & 154 & 92.8 & 366 & 96.3 & & \\
\hline \multirow[t]{4}{*}{ Thumb } & Right & No & 78 & 47 & 157 & 41.3 & 1.26 & $0.87-1.82$ \\
\hline & & Yes & 88 & 53 & 223 & 58.7 & & \\
\hline & Left & No & 67 & 40.4 & 135 & 35.5 & 1.23 & $0.85-1.79$ \\
\hline & & Yes & 99 & 59.6 & 245 & 64.5 & & \\
\hline \multirow[t]{4}{*}{ Interdigital } & Right & No & 34 & 20.5 & 42 & 11.1 & 2.07 & $1.26-3.40$ \\
\hline & & Yes & 132 & 79.5 & 338 & 88.9 & & \\
\hline & Left & No & 42 & 25.3 & 53 & 13.9 & 2.09 & $1.33-3.29$ \\
\hline & & Yes & 124 & 74.7 & 327 & 86.1 & & \\
\hline \multirow[t]{4}{*}{ Heel of the hand } & Right & No & 49 & 29.5 & 113 & 29.7 & 0.99 & $0.66-1.48$ \\
\hline & & Yes & 117 & 70.5 & 267 & 70.3 & & \\
\hline & Left & No & 54 & 32.5 & 117 & 30.8 & 1.08 & $0.73-1.60$ \\
\hline & & Yes & 112 & 67.5 & 263 & 69.2 & & \\
\hline \multirow[t]{4}{*}{ Back of the hand } & Right & No & 57 & 34.3 & 92 & 24.2 & 1.64 & $1.10-2.44$ \\
\hline & & Yes & 109 & 65.7 & 288 & 75.8 & & \\
\hline & Left & No & 42 & 25.3 & 65 & 17.1 & 1.64 & $1.06-2.55$ \\
\hline & & Yes & 124 & 74.7 & 315 & 82.9 & & \\
\hline \multirow{2}{*}{ Both hands } & & \multirow{2}{*}{ Value } & \multicolumn{2}{|c|}{ Male } & \multicolumn{2}{|c|}{ Female } & & 01050 \\
\hline & & & $\mathbf{N}$ & $\%$ & $\mathbf{N}$ & $\%$ & 年 & 年 \\
\hline \multirow[t]{2}{*}{ Palm } & & No & 17 & 10.2 & 21 & 5.5 & 1.95 & $0.99-3.79$ \\
\hline & & Yes & 149 & 89.8 & 358 & 94.5 & & \\
\hline \multirow[t]{2}{*}{ Thumb } & & No & 104 & 62.7 & 197 & 51.8 & 1.56 & $1.08-2.26$ \\
\hline & & Yes & 62 & 37.3 & 183 & 48.2 & & \\
\hline \multirow[t]{2}{*}{ Interdigital } & & No & 51 & 30.7 & 69 & 18.2 & 1.99 & $1.31-3.04$ \\
\hline & & Yes & 115 & 69.3 & 311 & 81.8 & & \\
\hline \multirow[t]{2}{*}{ Heel of the hand } & & No & 63 & 38 & 127 & 33.4 & 1.22 & $0.83-1.78$ \\
\hline & & Yes & 103 & 62 & 253 & 66.6 & & \\
\hline \multirow[t]{2}{*}{ Back of the hand } & & No & 67 & 40.4 & 128 & 33.7 & 1.33 & $0.92-1.94$ \\
\hline & & Yes & 99 & 59.6 & 252 & 66.3 & & \\
\hline \multicolumn{3}{|l|}{ Assessment - 4 categories } & & & & & תח & 1 \\
\hline Hand & & & $\mathbf{N}$ & $\%$ & $\mathbf{N}$ & $\%$ & (1) & 年 \\
\hline Right & & & 50 & 30.1 & 136 & 35.8 & 1 & - \\
\hline & & & 37 & 22.3 & 124 & 32.6 & 0.85 & $0.59-1.23$ \\
\hline & & & 49 & 29.5 & 70 & 18.4 & 1.53 & $1.11-2.11$ \\
\hline & & & 30 & 18.1 & 50 & 13.2 & 1.39 & $0.96-2.01$ \\
\hline Left & & & 55 & 33.1 & 155 & 40.8 & 1 & - \\
\hline & & & 53 & 31.9 & 116 & 30.5 & 1.19 & $0.87-1.64$ \\
\hline & & & 38 & 22.9 & 71 & 18.7 & 1.33 & $0.94-1.18$ \\
\hline & & & 20 & 12 & 38 & 10 & 1.31 & $0.86-2.00$ \\
\hline Both hands & & & 35 & 21.1 & 100 & 26.3 & 1 & - \\
\hline & & & 40 & 24.1 & 123 & 32.4 & 0.94 & $0.63-1.40$ \\
\hline & & & 49 & 29.5 & 88 & 23.2 & 1.37 & $0.96-1.98$ \\
\hline & & & 42 & 25.3 & 69 & 18.2 & 1.45 & $1.01-2.11$ \\
\hline
\end{tabular}


Table 1 - (continuation)

\begin{tabular}{|c|c|c|c|c|c|c|c|}
\hline \multirow{2}{*}{$\begin{array}{l}\text { Assessment - } 2 \text { categories } \\
\text { Hand }\end{array}$} & & \multicolumn{2}{|c|}{ Male } & \multicolumn{2}{|c|}{ Female } & \multirow{2}{*}{ OR } & \multirow{2}{*}{$\mathrm{Cl} 95 \%$} \\
\hline & & $\mathrm{N}$ & $\%$ & $\mathrm{~N}$ & $\%$ & & \\
\hline \multirow[t]{2}{*}{ Right } & Inappropriate & 79 & 47.6 & 120 & 31.6 & 1.97 & $1.35-2.86$ \\
\hline & Proper & 87 & 52.4 & 260 & 68.4 & & \\
\hline \multirow[t]{2}{*}{ Left } & Inappropriate & 58 & 34.9 & 109 & 28.7 & 1.33 & $0.90-1.97$ \\
\hline & Proper & 108 & 65.1 & 271 & 71.3 & & \\
\hline \multirow[t]{2}{*}{ Both hands } & Inappropriate & 91 & 54.8 & 157 & 41.3 & 1.72 & $1.19-2.49$ \\
\hline & Proper & 75 & 45.2 & 223 & 58.7 & & \\
\hline \multirow[t]{2}{*}{ Observations } & Some & 37 & 22.3 & 108 & 28.4 & 1.38 & $0.90-2.12$ \\
\hline & None & 129 & 77.7 & 272 & 71.6 & & \\
\hline
\end{tabular}

Table 2 - HAS spreading on students' hands as per nursing and medicine studies, year, sex, age and section. Facultad de Medicina de Badajoz. Badajoz. Spain. 2012/2014

\begin{tabular}{|c|c|c|c|c|c|c|c|}
\hline & \multirow{2}{*}{ Value } & \multicolumn{2}{|c|}{ Nursing } & \multicolumn{2}{|c|}{ Medicine } & \multirow{2}{*}{ OR } & \multirow{2}{*}{$\mathrm{Cl} 95 \%$} \\
\hline & & $\mathbf{N}$ & $\%$ & $\mathbf{N}$ & $\%$ & & \\
\hline \multirow[t]{2}{*}{ Year } & $2012 / 2013$ & 71 & 49.7 & 145 & 36.0 & 1.76 & $1.19-2.58$ \\
\hline & $2013 / 2014$ & 72 & 50.3 & 258 & 64.0 & & \\
\hline \multirow[t]{2}{*}{ Gender } & Male & 45 & 31.5 & 121 & 30.0 & 1.07 & $0.71-1.62$ \\
\hline & Female & 98 & 68.5 & 282 & 70.0 & & \\
\hline Age & Mean (years) & 21.2 & \pm 4.77 & 21.43 & \pm 3.2 & NS & \\
\hline \multirow{2}{*}{ Right Hand } & \multirow{2}{*}{ Value } & \multicolumn{2}{|c|}{ Nursing } & \multicolumn{2}{|c|}{ Medicine } & & \\
\hline & & $N$ & $\%$ & $\mathrm{~N}$ & $\%$ & Un & $6150 \%$ \\
\hline \multirow[t]{2}{*}{ Finger Tips } & No & 43 & 30.1 & 119 & 29.5 & 1.03 & $0.68-1.56$ \\
\hline & Yes & 100 & 69.9 & 284 & 70.5 & & \\
\hline \multirow[t]{2}{*}{ Back of the hand } & No & 48 & 33.6 & 101 & 25.1 & 1.51 & $0.99-2.28$ \\
\hline & Yes & 95 & 66.4 & 302 & 74.9 & & \\
\hline \multirow[t]{2}{*}{ Palm } & No & 11 & 7.7 & 8 & 2.0 & 4.12 & $1.62-10.45$ \\
\hline & Yes & 132 & 92.3 & 395 & 98.0 & & \\
\hline \multirow[t]{2}{*}{ Thumb } & No & 77 & 53.8 & 158 & 39.2 & 1.81 & $1.23-2.66$ \\
\hline & Yes & 66 & 46.2 & 245 & 60.8 & & \\
\hline \multirow[t]{2}{*}{ Between the fingers } & No & 47 & 32.9 & 29 & 7.2 & 6.31 & $3.78-10.56$ \\
\hline & Yes & 96 & 67.1 & 374 & 92.8 & & \\
\hline \multirow{2}{*}{ Left Hand } & \multirow{2}{*}{ Value } & \multicolumn{2}{|c|}{ Nursing } & \multicolumn{2}{|c|}{ Medicine } & OR & CI $95 \%$ \\
\hline & & $\mathbf{N}$ & $\%$ & $\mathbf{N}$ & $\%$ & Uก & 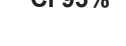 \\
\hline \multirow[t]{2}{*}{ Finger Tips } & No & 46 & 32.2 & 125 & 31.0 & 1.06 & $0.70-1.59$ \\
\hline & Yes & 97 & 67.8 & 278 & 69.0 & & \\
\hline \multirow[t]{2}{*}{ Back of the hand } & No & 39 & 27.3 & 68 & 16.9 & 1.85 & $1.18-2.90$ \\
\hline & Yes & 104 & 72.7 & 335 & 81.1 & & \\
\hline \multirow[t]{2}{*}{ Palm } & No & 9 & 6.3 & 17 & 4.2 & 1.53 & $0.66-3.50$ \\
\hline & Yes & 134 & 93.7 & 386 & 95.8 & & \\
\hline \multirow[t]{2}{*}{ Thumb } & No & 62 & 43.4 & 140 & 34.7 & 1.44 & $0.97-2.12$ \\
\hline & Yes & 81 & 56.6 & 263 & 65.3 & & \\
\hline \multirow[t]{2}{*}{ Between the fingers } & No & 40 & 28.0 & 55 & 13.6 & 2.46 & $1.58-3.90$ \\
\hline & Yes & 103 & 72.0 & 348 & 86.4 & & \\
\hline \multirow{2}{*}{ Both hands } & & & & & & & \\
\hline & value & N & $\%$ & N & $\%$ & Un & $6150 \%$ \\
\hline Finger Tips & No & 54 & 37.8 & 136 & 33.7 & 1.19 & $0.80-.77$ \\
\hline & Yes & 89 & 62.2 & 267 & 66.3 & & \\
\hline Back of the hand & No & 62 & 43.4 & 133 & 33.0 & 1.55 & $1.05-2.29$ \\
\hline & Yes & 81 & 56.6 & 270 & 67.0 & & \\
\hline Palms & No & 14 & 9.8 & 24 & 6.0 & 1.71 & $0.86-3.40$ \\
\hline & Yes & 129 & 90.2 & 378 & 94.0 & & \\
\hline
\end{tabular}


Table 2 - (continuation)

\begin{tabular}{|c|c|c|c|c|c|c|c|}
\hline \multirow[t]{2}{*}{ Thumbs } & No & 97 & 66.8 & 204 & 50.6 & \multirow[t]{2}{*}{2.06} & \multirow[t]{2}{*}{$1.38-3.07$} \\
\hline & Yes & 46 & 32.2 & 199 & 49.4 & & \\
\hline \multirow[t]{2}{*}{ Between the fingers } & No & 48 & 33.6 & 72 & 17.9 & \multirow[t]{2}{*}{2.32} & \multirow[t]{2}{*}{$1.51-3.57$} \\
\hline & Yes & 95 & 66.4 & 331 & 82.1 & & \\
\hline \multicolumn{2}{|l|}{ Assessment - 4 categories } & \multicolumn{2}{|c|}{ Nursing } & \multicolumn{2}{|c|}{ Medicine } & \multirow{2}{*}{ OR } & \multirow{2}{*}{$\mathrm{Cl} 95 \%$} \\
\hline Hand & 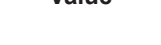 & $\mathbf{N}$ & $\%$ & $\mathbf{N}$ & $\%$ & & \\
\hline \multirow[t]{4}{*}{ Right } & Very good & 33 & 23.1 & 153 & 38.8 & 1 & - \\
\hline & Good & 38 & 26.6 & 123 & 30.5 & 1.34 & $0.88-2.03$ \\
\hline & Regular & 34 & 23.8 & 85 & 21.1 & 1.62 & $1.07-2.47$ \\
\hline & Bad & 38 & 26.6 & 42 & 10.4 & 2.7 & $1.84-3.98$ \\
\hline \multirow[t]{4}{*}{ Left Hand } & Very good & 45 & 31.5 & 165 & 40.9 & 1 & - \\
\hline & Good & 42 & 29.4 & 127 & 31.5 & 1.16 & $0.80-1.67$ \\
\hline & Regular & 32 & 22.4 & 77 & 19.1 & 1.37 & $0.92-2.02$ \\
\hline & Bad & 24 & 16.8 & 34 & 8.4 & 1.93 & $1.29-2.88$ \\
\hline \multirow[t]{4}{*}{ Both hands } & Very good & 20 & 14.0 & 115 & 28.5 & 1 & - \\
\hline & Good & 55 & 38.5 & 108 & 26.8 & 2.27 & $1.44-3.60$ \\
\hline & Regular & 13 & 9.1 & 124 & 30.8 & 0.64 & $0.33-1.13$ \\
\hline & Bad & 55 & 38.5 & 56 & 13.9 & 3.34 & $2.14-5.22$ \\
\hline \multicolumn{2}{|l|}{ Assessment - 2 categories } & \multicolumn{2}{|c|}{ Nursing } & \multicolumn{2}{|c|}{ Medicine } & \multirow{2}{*}{ OR } & \multirow{2}{*}{ Cl 95\% } \\
\hline Hand & 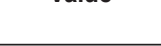 & $\mathbf{N}$ & $\%$ & $\mathbf{N}$ & $\%$ & & \\
\hline \multirow[t]{2}{*}{ Right } & Inappropriate & 72 & 50.3 & 127 & 31.5 & \multirow[t]{2}{*}{2.2} & \multirow[t]{2}{*}{$1.49-3.25$} \\
\hline & Proper & 71 & 49.7 & 276 & 68.5 & & \\
\hline \multirow[t]{2}{*}{ Left Hand } & Inappropriate & 56 & 39.2 & 111 & 27.5 & \multirow[t]{2}{*}{1.69} & \multirow[t]{2}{*}{$1.13-2.52$} \\
\hline & Proper & 87 & 60.8 & 292 & 72.5 & & \\
\hline \multirow[t]{2}{*}{ Both hands } & Inappropriate & 68 & 47.6 & 180 & 44.7 & \multirow[t]{2}{*}{1.12} & \multirow[t]{2}{*}{$0.77-1.65$} \\
\hline & Proper & 75 & 52.4 & 223 & 55.3 & & \\
\hline
\end{tabular}

Table 3 - Degree of HH performance in nursing and medical students' hands as per class, year, gender, age and section. Facultad de Medicina de Badajoz. Badajoz. Spain. 2012/2014

\begin{tabular}{|c|c|c|c|c|c|c|c|}
\hline & \multirow[t]{2}{*}{ Value } & \multicolumn{2}{|c|}{$\begin{array}{c}\text { HH } \\
\text { Inappropriate } \\
\end{array}$} & \multicolumn{2}{|c|}{$\begin{array}{l}\text { HH } \\
\text { Proper }\end{array}$} & \multirow[t]{2}{*}{ OR } & \multirow[t]{2}{*}{ Cl $95 \%$} \\
\hline & & $\mathbf{N}$ & $\%$ & $\mathbf{N}$ & $\%$ & & \\
\hline \multirow[t]{2}{*}{ Year } & $2012 / 2013$ & 128 & 51.6 & 88 & 29.5 & 2.55 & $1.79-3.62$ \\
\hline & $2013 / 2014$ & 120 & 48.4 & 210 & 70.5 & & \\
\hline \multirow[t]{2}{*}{ Course } & Nursing & 68 & 27.4 & 75 & 25.2 & 1.12 & $0.77-1.65$ \\
\hline & Medicine & 180 & 72.6 & 223 & 74.89 & & \\
\hline \multirow[t]{2}{*}{ Gender } & Male & 91 & 36.7 & 75 & 25.2 & 1.72 & $1.19-2.49$ \\
\hline & Female & 157 & 63.3 & 223 & 74.8 & & \\
\hline Age & Mean (years) & 21.18 & \pm 3.54 & 21.54 & \pm 3.88 & NS & \\
\hline \multirow[t]{2}{*}{ Right Hand } & & \multicolumn{2}{|c|}{$\begin{array}{c}\mathrm{HH} \\
\text { Inappropriate } \\
\end{array}$} & \multicolumn{2}{|c|}{$\begin{array}{c}\text { HH } \\
\text { Proper }\end{array}$} & OR & Cl $95 \%$ \\
\hline & & $\mathbf{N}$ & $\%$ & $\mathbf{N}$ & $\%$ & & \\
\hline \multirow[t]{2}{*}{ Finger Tips } & No & 116 & 46.8 & 46 & 15.4 & 4.81 & $3.22-7.19$ \\
\hline & Yes & 132 & 53.2 & 252 & 84.6 & & \\
\hline \multirow[t]{2}{*}{ Back of the hand } & No & 115 & 46.4 & 34 & 11.4 & 6.71 & $4.34-10.38$ \\
\hline & Yes & 133 & 53.6 & 264 & 88.6 & & \\
\hline \multirow[t]{2}{*}{ Palm } & No & 15 & 6 & 4 & 1.3 & 4.73 & $1.55-14.45$ \\
\hline & Yes & 233 & 94 & 294 & 98.7 & & \\
\hline \multirow[t]{2}{*}{ Thumb } & No & 179 & 72.2 & 56 & 18.8 & 11.21 & $7.50-16.56$ \\
\hline & Yes & 69 & 27.8 & 242 & 81.2 & & \\
\hline \multirow[t]{2}{*}{ Between the fingers } & No & 73 & 29.4 & 3 & 1 & 41.02 & $12.74-132.12$ \\
\hline & Yes & 175 & 70.6 & 295 & 99 & & \\
\hline
\end{tabular}


Table 3 - (continuation)

\begin{tabular}{|c|c|c|c|c|c|c|c|}
\hline \multirow[t]{2}{*}{ Left Hand } & & \multicolumn{2}{|c|}{$\begin{array}{c}\mathrm{HH} \\
\text { Inappropriate } \\
\end{array}$} & \multicolumn{2}{|c|}{$\begin{array}{c}\text { HH } \\
\text { Proper }\end{array}$} & \multirow[t]{2}{*}{ OR } & \multirow[t]{2}{*}{ CI $95 \%$} \\
\hline & & $\mathbf{N}$ & $\%$ & $\mathbf{N}$ & $\%$ & & \\
\hline \multirow[t]{2}{*}{ Finger Tips } & No & 120 & 48.4 & 51 & 17.1 & 4.54 & $3.07-6.71$ \\
\hline & Yes & 128 & 51.6 & 247 & 82.9 & & \\
\hline \multirow[t]{2}{*}{ Back of the hand } & No & 80 & 32.3 & 27 & 9.1 & 4.78 & $2.97-7.69$ \\
\hline & Yes & 168 & 67.7 & 271 & 90.9 & & \\
\hline \multirow[t]{2}{*}{ Palm } & No & 24 & 9.7 & 2 & 0.7 & 15.86 & $3.71-67.80$ \\
\hline & Yes & 224 & 90.3 & 296 & 99.3 & & \\
\hline \multirow[t]{2}{*}{ Thumb } & No & 161 & 64.9 & 41 & 13.8 & 11.6 & $7.62-17.66$ \\
\hline & Yes & 87 & 35.1 & 257 & 86.2 & & \\
\hline \multirow[t]{2}{*}{ Between the fingers } & No & 89 & 35.9 & 6 & 2 & 27.24 & $11.66-63.67$ \\
\hline & Yes & 159 & 64.1 & 292 & 98 & & \\
\hline \multirow{2}{*}{ Both hands } & & \multicolumn{2}{|c|}{$\begin{array}{c}\mathrm{HH} \\
\text { Inappropriate } \\
\end{array}$} & \multicolumn{2}{|c|}{$\begin{array}{c}\text { HH } \\
\text { Proper }\end{array}$} & OR & CI $95 \%$ \\
\hline & & $\mathbf{N}$ & $\%$ & $\mathbf{N}$ & $\%$ & & \\
\hline \multirow[t]{2}{*}{ Finger Tips } & No & 152 & 61.3 & 38 & 12.8 & 10.83 & $7.08-16.58$ \\
\hline & Yes & 96 & 38.7 & 260 & 87.2 & & \\
\hline \multirow[t]{2}{*}{ Back of the hand } & No & 155 & 62.5 & 40 & 13.4 & 10.75 & $7.06-16.37$ \\
\hline & Yes & 93 & 37.5 & 258 & 86.6 & & \\
\hline \multirow[t]{2}{*}{ Palms } & No & 34 & 13.7 & 4 & 1.3 & 11.64 & $4.07-33.29$ \\
\hline & Yes & 214 & 86.3 & 293 & 98.7 & & \\
\hline \multirow[t]{2}{*}{ Thumbs } & No & 219 & 88.3 & 82 & 27.5 & 19.89 & $12.51-31.62$ \\
\hline & Yes & 29 & 11.7 & 216 & 72.5 & & \\
\hline \multirow[t]{2}{*}{ Between the fingers } & No & 110 & 44.4 & 10 & 3.4 & 22.96 & $11.65-45.24$ \\
\hline & Yes & 138 & 55.6 & 288 & 96.6 & & \\
\hline
\end{tabular}

\section{Discussion}

$\mathrm{HH}$ is recognized globally as a key factor in the reduction of hospital-acquired infection occurrence. The WHO recommends that research and publications focus on the establishment of hydroalcoholic solution and assessment of its use via diverse strategies. Educational and awareness programs, workshops, reminder posters, direct observation to assess completion and adherence stand out among them ${ }^{(7,9)}$, as well as indirect assessment via proxy variables such as HAS use and hospital-acquired infection rates.

However, routine checking ${ }^{(10)}$ of methodology quality to improve $\mathrm{HH}$ adherence in order to reduce hospital-acquired infection is still inadequate to prove the efficiency of this approach; in addition to maintaining the biases in this type of study ${ }^{(11)}$.

Currently, the use of a motivational tool named positive deviation is suggested. This tool identifies groups of individuals that solve problems better than others without additional resources, which in a study conducted by Mara AR et al. (12) obtained an improvement, although no conclusive results were obtained in another routine revision ${ }^{(13)}$.

In another $\mathrm{HH}$ compliance study ${ }^{(14)}$ with interns in a Brazilian hospital, 50\% lower adherence was obtained, but this is no guarantee of performed handwashing efficiency via verification/assessment of proper $\mathrm{HH}$ technique. Likewise, nursing students had their internship in different hospitals, which prevented a follow-up; the introduction of this assessment in an undergraduate program becomes justified along with the five-step $\mathrm{HH}$ proposed by the $\mathrm{WHO}$, complete with adherence studies during the clinical internship and career.

There are few studies that assess the $\mathrm{HH}$ technique via marked HAS spreading. This is probably due to the $\mathrm{HH}$ guide provided by the $\mathrm{WHO}$ and other institutions that describe the solutions, their efficiency, and application sequence, but which do not provide statements about quality assessment.

Macdonald(15) assessed marked-HAS distribution in three sections (fingers, palms and thumbs) in trained staff, but the study does not detail the percentage of the sample who rubbed each individual section properly. In another study by the same author, the surface of a practice workshop was assessed before and after in the traumatology service, providing an estimate of the palm and back of the hand sections.

Widmer(16) found a great improvement and correlation between HAS covered areas scores and hand 
colony-forming units (CFUs), before and after specific training, which was compulsory for the staff.

Hautmaniere $^{(17)}$ and Sutter(18) performed a beforeafter assessment of specific $\mathrm{HH}$ training programs for medical students, improving sections covered with HAS and CFU spreading; they concluded that this tool is easy and trustworthy for gauging the $\mathrm{HH}$ technique.

$K$ ampf ${ }^{(19)}$ found that $53 \%$ of subjects studied left out at least one section during $\mathrm{HH}$, using the reference technique in the EN1500 norm; although the sample was small (55 people) and had many comparisons (16 variables).

Via a compulsory educational course, Szilágy(20) obtained an assessment of $67-72 \%$ from 4642 participants with a "good" notation; in that study, the sections forgotten most frequently were the top section of the fingers close to the nails, the thenar eminence, and the wrist. These results are similar to the present study, although this last one was performed on students and was voluntarily.

In Spain, only the study conducted by RamonCanton $^{(21)}$ assessed $\mathrm{HH}$ technique in healthcare professionals at their work post, with no previous compulsory workshop. The results showed that $95.2 \%$ of people assessed left at least one section unclean, and the sections with the worst scores were the thumbs and fingers. In our study, the same assumption gives a result of $75.27 \%$ with at least one section of the hand left unclean, and the sections with worst scoring were the thumbs and in between the fingers.

Other studies ${ }^{(17,22)}$ involving medical and nursing students obtained a rating of inadequate HAS $\mathrm{HH}$ of $78.5 \%$ and $81.5 \%$, much higher than our study (49.82\%).

Furthermore, $26.6 \%$ of the students were observed to have attended the practicum with long nails, with nail polish or artificial nails, watches or bracelets; these circumstances complicate correct $\mathrm{HH}$ performance, and were not taken into account in other studies.

It is important to point out that the right hand on its own was better cleaned with HAS than the left one, except the thumb; considering that most of the human population is right-handed, this entails that the dominant hand is washed less properly. Therefore, emphasis should be placed on raising awareness and training the non-dominant hand on $\mathrm{HH}$.

Likewise, comments and questions of the students attending were heeded, this helped identify the fact that they had difficulty in recognizing the opportunities for $\mathrm{HH}$ according to the different procedures that form their usual clinical practice. All these elements must be taken into account and incorporated into cross-disciplinary education during undergraduate studies.

Knowledge that health care students must have about hand, object and surface contamination and $\mathrm{HH}$ issues in hospital-acquired infection prevention and control is key to improve $\mathrm{HH}$ quality and adherence ${ }^{(23-24)}$ to provide safe health services.

\section{Conclusions}

All staff in a health institution, and specially heath care professionals, including students during their internship, must deliver safe health services that prevent hospital-acquired infection in their everyday practice.

Therefore, proper education and training in proper $\mathrm{HH}$ technique performance and regular creation of campaigns and workshops remains a priority.

Moreover, effectiveness of $\mathrm{HH}$ also depends on quality technique, and we believe that regular practicum and assessment using this immediate feedback method could provide a simple, quick tool with large effect in students and professionals; it can ascertain $\mathrm{HH}$ technique quality at an individual level, after a course/workshop or at their place of work, giving them the necessary skills and knowledge as well as awareness and better adherence, which need improvement.

Hand hygiene improvement must be a priority for healthcare authorities in all levels, be it undergraduate, graduate studies or ongoing training, where there is an individual responsibility for each healthcare professional. All $\mathrm{HH}$ programs must include different actions, such as alcoholic solution introduction, staff education and motivation, as well as assessment and counselling in $\mathrm{HH}$ technique quality.

\section{References}

1. Pittet D, Allegranzi B, Boyce J. The World Health Organization Guidelines on Hand Hygiene in Health Care and their consensus recommendations. Infect Contro Hosp Epidemiol. 2009 Jul;30(7):611-22.

2. Allegranzi $B$, Pittet D. Role of hand hygiene in healthcare-associated infection prevention. J Hosp Infect. 2009 Dec;73(4):305-15.

3. Creedon SA. Healthcare workers' hand decontamination practices: compliance with recommended guidelines. J Adv Nurs. 2005 Aug;51(3):208-16.

4. Chou DTS, Achan P, Ramachandran M. The World Health Organization " 5 moments of hand hygiene": 
the scientific foundation. J Bone Joint Surg Br. 2012 Apr;94(4):441-5.

5. Ministerio de Sanidad, Servicios Sociales e Igualdad. Plan de Calidad del Sistema Nacional de Salud. Fomentar la excelencia clínica. Estrategia 8.- Mejorar la seguridad de los pacientes atendidos en los centros sanitarios del SNS. [Internet]. [acesso 25 ago 2014]; Disponível em: http://www.msssi.gob.es/organizacion/ sns/planCalidadSNS/ec03_doc.htm.

6. Junta de Extremadura (ES). Consejería de Sanidad y Dependencia. Servicio Extremeño de Salud. Plan Estratégico de Seguridad de Pacientes del Servicio Extremeño de Salud 2011-2016 [Internet]. Cáceres, 2011 [acesso 25 ago 2014]. Disponível em: http:// www.msssi.gob.es/organizacion/sns/planCalidadSNS/ ec03_doc.htm

7. Erasmus V, Daha TJ, Brug H, Richardus JH, Behrendt MD, Vos MC, et al. Systematic review of studies on compliance with hand hygiene guidelines in hospital care. Infect Control Hosp Epidemiol. 2010 Mar;31(3):283-94. 8. Gerencia del Área de Salud de Badajoz (ES). Servicio Extremeño de Salud. Memoria de Área de Salud de Badajoz [Internet]. 2012 [acesso 25 ago 2014]. Disponível em: http://www.areasaludbadajoz.com/ index.php/component/flexicontent/11-atencion-alusuario/17-memorias-anuales.

9. Rosenthal VD, Pawar M, Leblebicioglu $H$, Navoa-Ng JA, Villamil-Gómez W, Armas-Ruiz A, et al. Impact of the International Nosocomial Infection Control Consortium (INICC) Multidimensional Hand Hygiene Approach over 13 Years in 51 Cities of 19 Limited-Resource Countries from Latin America, Asia, the Middle East, and Europe. Infect Control Hosp Epidemiol. 2013;34(4):415-23.

10. Gould DJ, Moralejo D, Drey N, Chudleigh JH. Interventions to improve hand hygiene compliance in patient care. Cochrane Database of Systematic Reviews 2010, Issue 9. Art. No.: CD005186. DOI: 10.1002/14651858.CD005186.pub3.

11. Kohli E, Ptak J, Smith R, Taylor E, Talbot EA, Kirkland $K B$. Variability in the Hawthorne Effect With Regard to Hand Hygiene Performance in High- and Low-Performing Inpatient Care Units. Infect Control Hosp Epidemiol. 2009 Mar;30(3):222-5.

12. Marra AR, Noritomi DT, Westheimer Cavalcante AJ, Sampaio Camargo TZ, Bortoleto RP, Durao Junior MS, et al. A multicenter study using positive deviance for improving hand hygiene compliance. Am J Infect Control. 2013; 41:984-8.

13. Cherry MG, Brown JM, Bethell GS, Neal T, Shaw

NJ. Features of educational interventions that lead to compliance with hand hygiene in healthcare professionals within a hospital care setting. A BEME systematic review: BEME Guide No. 22. Med Teach. 2012;34(6):e406-20. doi: 10.3109/0142159X.2012.680936.

14. Felix CC, Miyadahira AM. Evaluation of the handwashing technique held by students from the nursing graduation course. Rev Esc Enferm USP. 2009 Mar;43(1):139-45.

15. Macdonald DJM, McKillop ECA, Trotter S, Gray AJ. Improving hand-washing performance - a crossover study of hand-washing in the orthopaedic department. Ann R Coll Surg Engl. 2006 May;88(3):289-91.

16. Widmer AF, Conzelmann $M$, Tomic $M$, Frei $R$, Stranden AM. Introducing alcohol-based hand rub for hand hygiene: the critical need for training. Infect Control Hosp Epidemiol. 2007 Jan;28(1):50-4.

17. Hautemaniere A, Diguio N, Daval MC, Hunter PR, Hartemann P. Short-term assessment of training of medical students in the use of alcohol-based hand rub using fluorescent-labeled hand rub and skin hydration measurements. Am J Infect Control. 2009 May;37(4):338-40.

18. Tschudin Sutter S, Frei R, Dangel M, Widmer AF. Effect of teaching recommended World Health Organization technique on the use of alcohol-based hand rub by medical students. Infect Control Hosp Epidemiol. 2010 Nov;31(11):1194-5.

19. Kampf G, Reichel M, Feil Y, Eggerstedt S, Kaulfers $\mathrm{P}-\mathrm{M}$. Influence of rub-in technique on required application time and hand coverage in hygienic hand disinfection. BMC Infect Dis. 2008;8:149.

20. Szilagyi L, Haidegger T, Lehotsky A, Nagy M, Csonka $\mathrm{E}-\mathrm{A}$, Sun $\mathrm{X}$, et al. A large-scale assessment of hand hygiene quality and the effectiveness of the "WHO 6-steps". BMC Infect Dis. 2013;13:249.

21. Ramon-Canton C, Boada-Sanmartin N, PagespetitCasas L. [Evaluation of a hand hygiene technique in healthcare workers]. Rev Calid Asist Organo Soc Espanola Calid Asist. 2011 Dec;26(6):376-9.

22. Kelcikova S, Skodova Z, Straka S. Effectiveness of hand hygiene education in a basic nursing school curricula. Public Health Nurs Boston Mass. 2012 Apr;29(2):152-9.

23. Molina-Cabrillana J, Alvarez-Leon EE, Quori A, Garciade Carlos P, Lopez-Carrio I, Bolanos-Rivero M, et al. [Assessment of a hand hygiene program on healthcareassociated infection control]. Rev Calid Asist Organo Soc Espanola Calid Asist. 2010 Aug;25(4):215-22.

24. Garcia-Vazquez E, Murcia-Paya J, Allegue JM, Canteras M, Gomez J. [Influence of a multiple 
intervention program for hand hygiene compliance in an ICU]. Med Intensiva Soc Espanola Med Intensiva Unidades Coronarias. 2012 Mar;36(2):69-76. 